\title{
A mutant of Metarhizium anisopliae var. acridum with enhanced submerged conidiation
}

\author{
Peter W. Inglis, Rubia B.C. Sarmento, Camila F.C. Gavião, Heloisa Frazão and M. Cléria Valadares-Inglis \\ Embrapa Recursos Genéticos e Biotecnologia, Parque Estação Biológica, Final Av. W/5 Norte, C.P. 02372, CEP- \\ 70770900, Brasília-DF, Brazil \\ *Author for correspondence: Tel.: +55-61-4484784,Fax: +55-61-4484673, E-mail: peteringlis@onetel.com
}

Received 25 April 2005; accepted 3 June 2005

Keywords: filamentous fungi, Metarhizium anisopliae var. acridum, submerged conidiation, sporulation

\section{Summary}

An insertional mutant of Metarhizium anisopliae is described with enhanced submerged conidiation. In a $500 \mathrm{ml}$ submerged culture, this mutant produces a mean of $4.05 \times 10^{8}$ propagules $\mathrm{ml}^{-1}$ from an inoculum of $1 \times 10^{6}$ conidia, where the parental strain accumulates only $3.75 \times 10^{4}$ propagules $\mathrm{ml}^{-1}$.

The mitosporic Hyphomycete fungus, Metarhizium anisopliae var. acridum is of considerable interest as a specific biocontrol agent for locusts and grasshoppers. A Brazilian isolate, M. anisopliae var. acridum CG423, was found to cause high mortality in local pest species of grasshoppers and is currently being developed in Brazil as a biological pesticide (Magalhães et al. 2001). As part of the further development and improvement of this strain, we previously reported its co-transformation with genes for green fluorescent protein (egfp) and resistance to gluphosinate ammonium (bar) (Inglis et al. 2000). From among these co-transformants, we later observed a strain, CG819, showing abundant submerged conidiation in deep shake-flask cultures. The culture medium of this strain became a deep green color within $96 \mathrm{~h}$, due to the accumulation of pigmented conidia (Figure 1a); producing a mean of $4.05 \times 10^{8}$ propagules $\mathrm{ml}^{-1}$ in triplicate flasks $(500 \mathrm{ml}$ medium in $1000 \mathrm{ml}$ conical flask). Under the same conditions, the culture medium of the wild-type parental strain, CG423 accumulated a mean of $3.75 \times 10^{4}$ propagules $\mathrm{ml}^{-1}$ and another bar/ egfp co-transformant of this strain, CG820, produced $3.5 \times 10^{5}$ propagules $\mathrm{ml}^{-1}$. Under conditions of submerged conidiation, the mycelial phase of CG819 appears to be severely restricted. The cultures appear heterogeneous, where spores appear to be budded directly from other unicellular propagules or be produced from slightly elongated phialide-like structures, which appeared to be free or as branches of stunted hyphae (arrowed, Figure 1b). In contrast, the morphology of CG820 in deep shake flask cultures is wild-type (Figure 1c); appearing as clusters or pellets of elongated, non-pigmented mycelial hyphae.

Slide cultures were set up to observe conidiation within agar. Strain CG819 was found to conidiate readily within the agar layer (Figure 2a). In contrast, conidiation within the agar blocks appeared to be totally suppressed in wild-type parental strain, CG423 and the control co-transformant, CG820 (Figure 2b). No significant differences in the appearance of normal aerial conidiation at the exposed edges of the slide cultures were observed between the three strains. The embedded conidia of CG819 appeared to be produced in coiled or bundle-like structures in the agar milieu (Figure 2c). This appearance is probably a result of the normal conidiation process in this fungus, where phialoconidia are produced in terminal chains.

While some strains of Metarhizium have been reported to be able to produce submerged conidia under certain culture conditions (Fargues et al. 2001), the genetic control of this character and its high variability among strains is unknown. Since strain CG423 normally sporulates poorly in submerged culture, our results suggest that the genome of co-transformant CG819, probably carries an insertion of one of the marker genes in a locus that regulates this characteristic. This mutant 


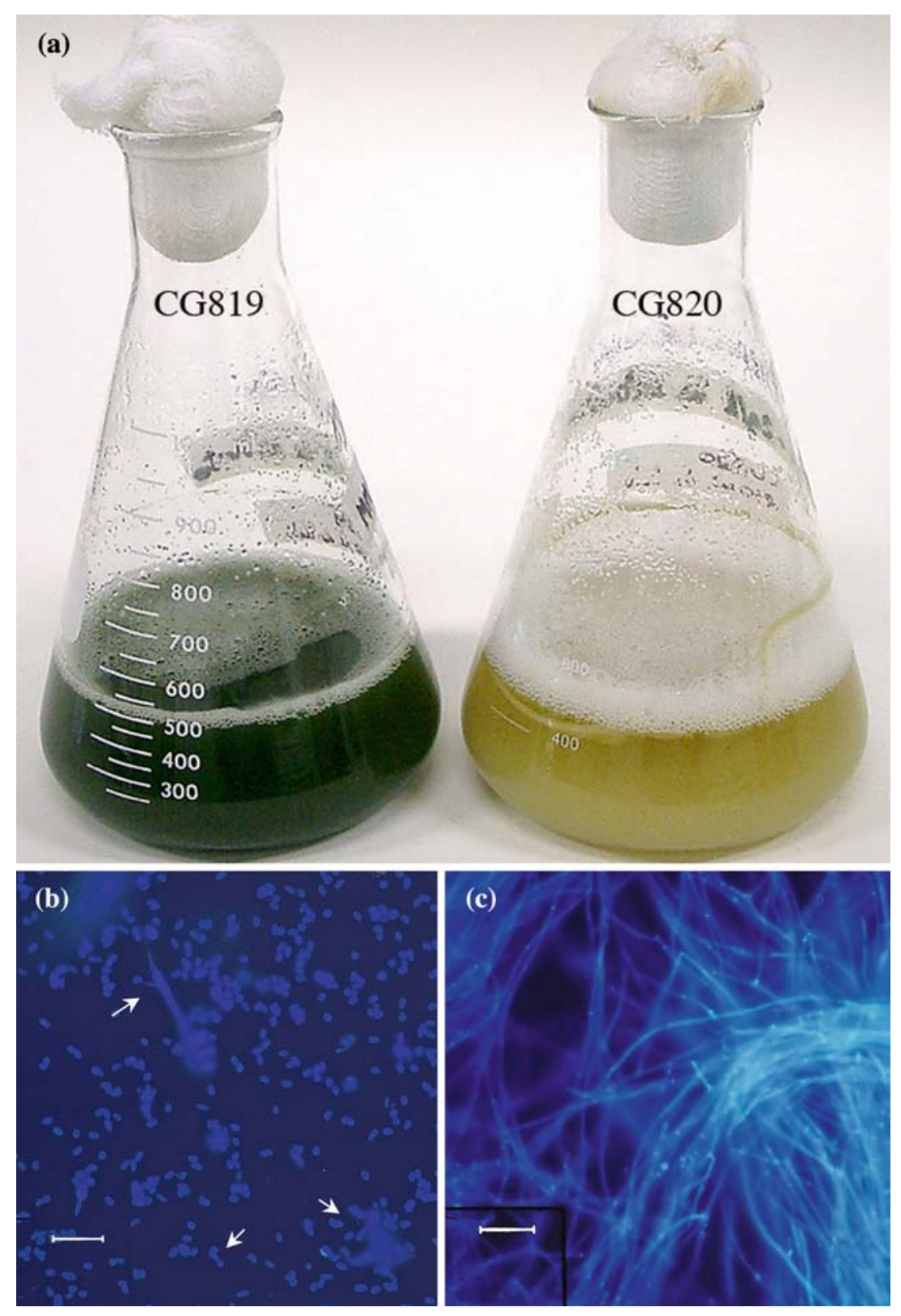

Figure 1. Submerged conidiation in a shake flask culture of $M$. anisopliae var. acridum insertional mutant CG819, compared with co-transformant CG820, carrying the same marker genes (Panel a). One litre Erlenmeyer flasks containing $500 \mathrm{ml}$ Aspergillus minimal medium with Hutner's trace elements, supplemented with $2.0 \mathrm{~g} \mathrm{l}^{-1}$ yeast extract, were inoculated with $1 \times 10^{6}$ conidia and incubated for $96 \mathrm{~h}$ at $28{ }^{\circ} \mathrm{C}$ with shaking at $200 \mathrm{rpm}$. Panels $\mathrm{b}$ and c show the general aspect of submerged cultures of CG819 and CG820 respectively, stained with the fluorescent dye, Tinopal $($ bar $=20 \mu \mathrm{m})$.

should therefore be useful in the molecular genetic analysis of submerged conidiation in Metarhizium. Such data may then permit the directed mutagenesis of other strains, permitting a wider assessment of submerged conidiation as an alternative method for the bulk production of infective propagules in strains normally lacking this characteristic.

\section{Acknowledgements}

The authors wish to thank The Brazilian National Research Council, CNPq for a postdoctoral bursary for PWI. A patent has been applied by Embrapa for mutant strain CG819 of M. anisopliae var. acridum, registered under number 000175 . 

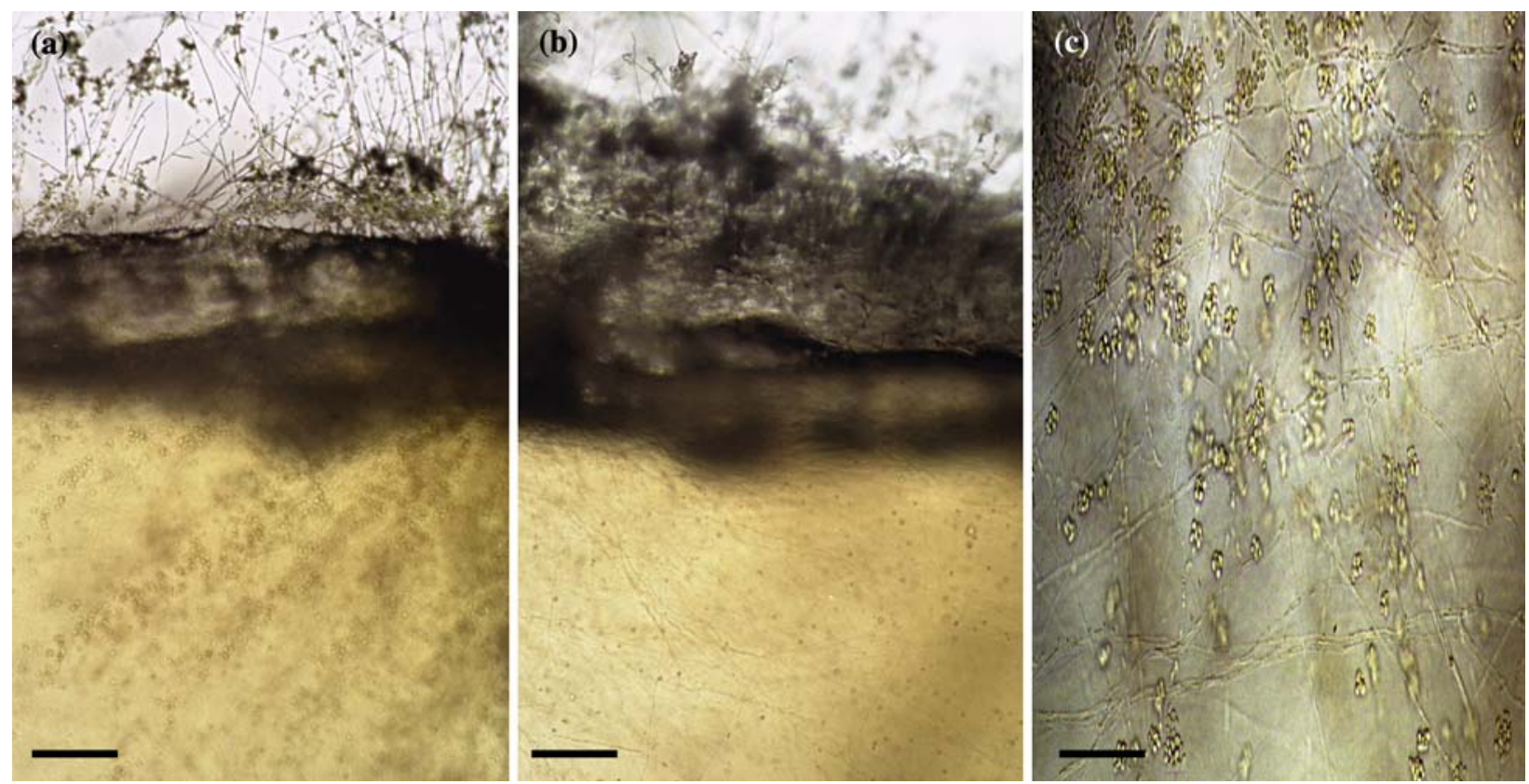

Figure 2. Slide cultures of $M$. anisopliae var. acridum insertional mutant, CG819 (Panels a; bar $=250 \mu \mathrm{m}$ and c; bar $=50 \mu \mathrm{m}$ ), compared with cotransformant CG820 (Panel b; bar $=250 \mu \mathrm{m})$. Sections $(1.5 \times 1.5 \times 0.5 \mathrm{~cm})$ of solidified potato dextrose agar were placed on sterile microscope slides and a sterile cover slip placed over the agar. Each of the four exposed, $0.5 \mathrm{~cm}$ deep, faces of agar were then inoculated with conidia and incubated at $28^{\circ} \mathrm{C}$ for 5 days.

\section{References}

Fargues, J., Smits, N., Vidal, C., Vey, A., Vega, F., Mercadier, G. \& Quimby, P. 2001 Effect of liquid culture media on morphology, growth, propagule production, and pathogenic activity of the Hyphomycete, Metarhizium flavoviride. Mycopathologia 154, 127138.

Inglis, P.W., Aragão, F.J.L., Frazão, H., Magalhães, B.P \& ValadaresInglis, M.C. 2000 Biolistic co-transformation of Metarhizium ani- sopliae var. acridum strain CG423 with green fluorescent protein and resistance to glufosinate ammonium. FEMS Microbiology Letters 191, 249-254.

Magalhaes, B.P., Faria, M.R., Lecog, M., Schimidt, F.G.V., Silva, J.B.T. \& Frazao, H.S 2001 The use of Metarhizium anisopliae var. acridum against the grasshopper Rhammatocerus schistocercoides in Brazil. Journal of Orthoptera Research 10, 199-202. 